López González, D. y Gerrúdix-Barrio, F. La magia como recurso didáctico-lúdico para el desarrollo de la creatividad en niñas y niños de 5 años. Aula de Encuentro, n² 21, volumen 1, pp. 143-159

\title{
LA MAGIA COMO RECURSO DIDÁCTICO-LÚDICO PARA EL DESARROLLO DE LA CREATIVIDAD EN NIÑAS Y NIÑOS DE 5 AÑOS
}

\section{MAGIC AS A DIDACTIC-PLAYFUL RESOURCE FOR THE DEVELOPMENT OF THE CREATIVITY IN 5-YEAR-OLDS}

\section{David López González}

Felipe Gértrudix-Barrio

\begin{abstract}
Resumen
La magia, por su carga lúdica, por su naturaleza abierta y grado de representación, ofrece un maravilloso campo de trabajo que, desde la dimensión educativa, debe aprovecharse como recurso de primer orden, en especial en los primeros años. En este sentido, el trabajo tiene por objetivo comprobar la eficiencia de la magia como recurso educativo en el proceso de enseñanza-aprendizaje entre los estudiantes de educación infantil. Para ello, se ha llevado a cabo una investigación-acción en un aula de cinco años de educación infantil utilizando como instrumentos de investigación; la entrevista en profundidad a expertos y docentes en magia y educación y la rúbrica de evaluación en la observación directa e intervención realizada en el aula. Se ha comprobado que la magia tiene un valor positivo para desarrollar una serie de habilidades relacionadas con la creatividad fomentando en el alumno una mejor predisposición por aprender.
\end{abstract}




\title{
Palabras Clave
}

magia, creatividad, educación infantil, investigación-acción, entrevista en profundidad, rúbrica de evaluación

\begin{abstract}
Magic, because of its ludic charge, because of its open nature and degree of representation, offers a wonderful field of work that, from the educational dimension, should be used as a resource of the first order, especially in the first years. In this sense, the work aims to verify the efficiency of magic as an educational resource in the teaching-learning process among children in early childhood education. To this end, an action research has been carried out in a classroom of five years of early childhood education using research instruments; the in-depth interview with experts and teachers in magic and education and the rubric of evaluation in direct observation and intervention carried out in the classroom. It has been proven that magic has a positive value to develop a series of skills related to creativity, fostering in the student a better predisposition to learn.
\end{abstract}

\section{Key Words}

magic, creativity, childhood education, action research, in-depth interview, assessment rubric.

\section{Introducción}

Tener la posibilidad de acceder a recursos en cualquier aula de educación infantil que provoquen una mejora del proceso de enseñanza-aprendizaje, debería ser fundamental y respaldada por todos los agentes educativos implicados. Pero esta situación no es evidente en todos los centros, aulas, ni en todos los docentes. Los recursos, tanto materiales como personales, han de ser una herramienta eficaz que facilite al maestro a encontrar metodologías idóneas y personalizadas que favorezcan el aprendizaje en el niño; es decir, disponer de una "bolsa" donde en su interior estén guardados "sus recursos": el teatro, la música, los recursos materiales, la danza, las tecnologías, el guiñol, la papiroflexia o la magia, entre otros, con el fin de convertirse en cantante, bailarín, actor, payaso, mago, experto en tecnologías, etc. En definitiva, ser un docente del siglo $\mathrm{XXI}$, innovador con gran capacidad de adaptación: un maestro polivalente 
capaz de desarrollar actividades lúdicas, motivadoras e innovadoras para el alumno (Ruíz, 2013).

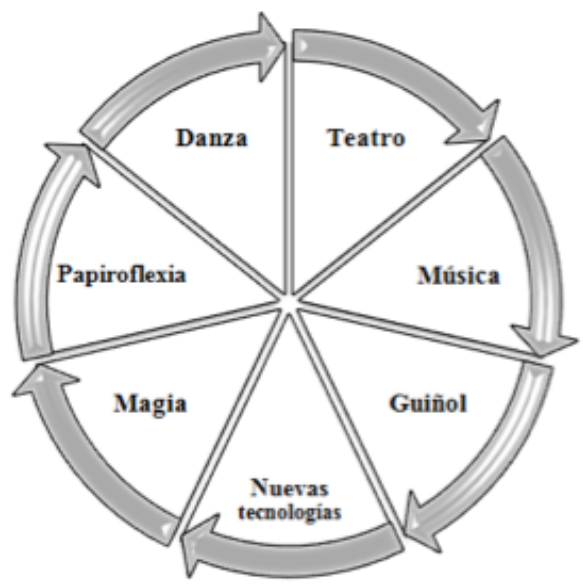

Figura 1. Recursos para trabajar en el aula. Fuente: elaboración propia

Entre estos recursos nos encontramos con la magia; con una carga lúdica implícita que, por su naturaleza abierta y grado de representación, ofrece un maravilloso campo de trabajo que, desde la dimensión educativa, debe aprovecharse (Gértrudix \& Gértrudix, 2013). Existen precedentes exitosos de su utilización educativa en España, como es el caso del maestro Xuso Ruíz (premio al mérito educativo en 2015, en la Comunidad Autónoma de Andalucía), quien lo ha utilizado para el aprendizaje de las matemáticas en educación primaria. El proyecto Informagia del colegio Alameda de Osuna de Madrid, a través del profesor Juan Morata Sanz (Educación 3.0, 2014), ha buscado que los estudiantes de $2^{\circ}$ y $3^{\circ}$ curso de primaria adquirieran competencias audiovisuales y digitales (Escuela de Magia Alameda, 2014). En el ámbito de la educación infantil descubrimos el trabajo de Raquel Simó, quien realizó una investigación con alumnos de 4 años en un colegio de la ciudad de Zamora (Simó, 2014). En otras latitudes geográficas encontramos a Jody Greig, profesor de matemáticas en un centro de secundaria de Escocia, el cual ha publicado diversos trabajos y recursos en torno a la Magia y a la aritmética en una colección denominada Entertainer Hub (Greig, 2016).

Es por ello, que antes de llevar a cabo nuestra investigación se formularon una serie de interrogantes: ¿puede ser un maestro mago?, ¿qué factores de la creatividad se pueden desarrollar con la magia en educación infantil?, ¿es la magia un recurso didáctico y lúdico viable en uno de educación infantil?

\section{Marco teórico}

\subsection{La magia como recurso educativo y lúdico.}

No cabe duda de que la magia como recurso educativo y lúdico es fruto de una metodología basada en la participación del alumnado. Estos referentes metodológicos ya los encontramos en las ideas promulgadas por Rousseau a las ideas definidas en la escuela nueva que sitúan al niño como protagonista en su aprendizaje (Otero, 2000; 
Paquette, 1976); en las propuestas declaradas por María Montessori, al poner al alcance del niño los medios necesarios para su desarrollo espontáneo, autoeducación y autonomía (Codoni, 1987).

Por otro lado, Dewey y Decroly anticiparon el "to learn by loving" como "proyectos de investigación" que sitúan el aprendizaje de trabajos dentro de la escuela y que son significativos fuera de ella. Por último, y como colofón de esa evolución de las metodologías activas, debemos aludir a Freinet (1973) quien apela la labor del maestro a preparar y ofrecer un ambiente, un material y unas técnicas que permitan una educación natural para la convivencia y la vida social (Vidal, 2009). Es por ello, que la magia, como recurso didáctico alberga un gran potencial aún por explorar adecuadamente en el aula: es un recurso que ayuda a entender procesos complejos en la ciencia, la tecnología, las matemáticas o la ingeniería, y por supuesto: un espectáculo de magia bien diseñado puede ser muy educativo, ya que es entretenido. Realizando simples trucos se podrá trabajar el área lógico-matemática, aumentar el vocabulario y fomentar la adquisición del lenguaje escrito o potenciar los buenos comportamientos; en definitiva, el pensamiento mágico aumenta la creatividad, "sembrando la ilusión del que se asoma a un mundo en el que todo puede ser posible" (Martín, 2011).

Teniendo en cuenta estos anclajes teóricos, la magia como recurso educativo cobra protagonismo e importancia. El uso de la magia en el aula de educación infantil debe llevarse a cabo mediante una metodología que esté asentada en la actividad y en la creatividad, desde una triple perspectiva: a) actividades creativas, b) actividades de efectuación y c) actividades de fabricación (Amegan, 1993).

\subsection{Magia y creatividad}

Podemos decir, de manera general, que la creatividad acompaña a aquellas personas que están dotadas de ciertas características, como son: a) capaz de definir ideas novedosas (Engle, 1997), en las que se incluyan elementos de novedad (Davis, 1983), y una gran intuición para resolver problemas (Bransford, 1984), equilibrando el análisis, la inventiva y la práctica (Sternberg, 1997). En definitiva, la creatividad es un "rasgo complejo en el que influyen tantos procesos cognitivos, motivacionales y socioculturales" (Sanz de Acedo Lizárraga y Sanz de Acedo Baquedano, 2007, pág. 16).

Comentábamos más arriba, que la magia como recurso educativo lleva consigo una metodología activa, participativa y basada en la creatividad. A esta afirmación, hemos de añadir que actúa con una gran motivación intrínseca. Por sí misma lleva una carga creativa que proporciona disfrute. $E$ incluso, desarrollando la magia (no como espectador sino siendo responsable de llevarlo a la práctica) da la oportunidad al estudiante de tomar sus propias decisiones, de estar seguro de sí mismo y decidir de forma consciente continuar su camino hasta llegar a conseguir su meta, disfrutando a la vez que aprende y, quizás lo más importante, de forma voluntaria. Así pues, en este caso, estamos ante una persona creativa.

Robinson (2011), experto en creatividad, la define a través de tres conceptos clave para él: a) la imaginación, la creatividad y la innovación. 
La creatividad implica lo que algunos científicos resumen como aprender algo nuevo, realizar conexiones o comparaciones con información diferente y demostrar talento. Storr (1988) describe tres etapas en la vida de una persona creativa:

a) Aprende lo que ha estado investigando y emplea el necesario tiempo imitando palabras y acciones de otros.

b) Un momento de destreza y de reflexión mejorando su comunicación en un campo específico.

c) Se aleja del campo, explora ideas abstractas, inventa, reflexiona, produce trabajos y tiene un estilo de vida acorde a su producción.

Por esta razón, sabemos que mediante la metodología creativa se desarrollan varios tipos de creatividad que se interrelacionan entre sí: a) expresiva, b) productiva, c) inventiva, d) innovadora, y e) emergente (Sanz de Acedo Lizárraga y Sanz de Acedo Baquedano, 2007).

Al igual que con la motivación, se destaca el desarrollo de la creatividad mediante la pasión, la devoción (Goleman, 1995), y la persistencia (Newell, 1962).

Haciendo uso de la magia como elemento motivador y desarrollador de su creatividad, el estudiante emplea la pasión ante el juego, la devoción ante lo que espera y la persistencia al ponerlo en práctica. Todo ello no podría alcanzarse, si no se emplea una adecuada enseñanza creativa, ya sea mediante una técnica, un procedimiento o una estrategia.

Según Smith (1970) existen una serie de principios dentro de una enseñanza creativa y no se duda que todos ellos tienen cabida dentro de una enseñanza con magia. Estos son los siguientes: 1) se espera del alumno resultados nuevos, diferentes o únicos, 2) presta tanta importancia al proceso creativo como al propio producto creativo, 3) estimula el pensamiento divergente, 4) reconoce la importancia de ciertas condiciones: intelectuales, físicas, socioemocionales, psicológicas y pedagógicas en el desarrollo del pensamiento creativo, 5) parte de una tensión motivante de los alumnos y permite reducir dicha tensión, 6 ) insiste en el éxito logrado por los alumnos y no en sus fracasos, 7) utiliza situaciones abiertas de aprendizaje, 8) promueve conocimientos, pero también prevé la utilización de dichos conocimientos en situaciones de resolución de problemas, 9) permite, de cuando en cuando, que el alumno se enfrente solo a lo desconocido, 10) alienta la evaluación y la crítica constructiva, 11) alienta la evaluación y la crítica constructiva, 12) da lugar a resultados a menudo imprevisibles, 13) impulsa a la manipulación y exploración de los objetos y de las ideas, 14) permite la utilización del pensamiento "preconsciente" (experiencias personales, emociones personales"), 15) utiliza el proceso democrático haciendo participar al alumno en la vida del grupo, 16) utiliza métodos adecuados para el desarrollo del pensamiento creativo, 17) incita a los alumnos a generar y desarrollar sus propias ideas, 18) insiste en las diferencias individuales, en la unicidad y originalidad de los alumnos.

Dado que nuestro objetivo es emplear la magia como un recurso para desarrollar la creatividad, éste se ha considerado como un modelo adecuado el elaborado por 
Williams (1970), que defiende la interacción entre el alumno y el educador distinguiendo tres dimensiones:

a) Las materias por impartir o por aprender.

b) Las estrategias del educador para hacer reaccionar al alumno en el aprendizaje de dichas materias.

c) Los tipos de comportamientos o respuestas del alumno.

Pero ¿cómo debe realizarse para que se desenvuelva de manera creativa? Una metodología creativa debe contener una enseñanza abierta y flexible. Para ello describe las habilidades que el alumno desarrolla o puede desarrollar con este modelo, ya sean afectivas o cognoscitivas como la facilidad para generar muchas ideas, originales y nuevas, que sean únicas y provechosas y la facilidad para detallar y desarrollar las propias ideas. Entre ellas, resalta: 1) el amor al riesgo, 2) el amor a la complejidad, y 3) la imaginación (Williams, 1970).

Por otra parte, las estrategias que emplea el educador pueden condicionar la del alumno. Se ha comprobado científicamente que, si el maestro es directivo o muy estructurado, los comportamientos de los alumnos son similares; y, cuando los requerimientos del maestro son más abiertos, los alumnos se comportan de manera abierta y creativa. El maestro que quiera conseguir este comportamiento creativo por parte de sus alumnos ha de emplear estrategias propiamente creativas donde destaque la participación del alumno mediante, o bien, preguntas provocadoras como ¿qué pensáis que haríamos ahora si nuestra clase estuviera al aire libre?, investigaciones a partir de una situación dada, comparar palabras con una concreta, o bien actividades que requiera cualquiera de las habilidades anteriormente nombradas.

\section{Método}

\subsection{Objetivos}

El trabajo da respuesta a una serie de objetivos planteados a través de una investigación-acción (Login, 2004), realizada durante el curso 2015-2016 con estudiantes de cinco años de educación infantil en un C.E.I.P de la provincia de Toledo. Estos objetivos son:

1. Comprobar si existe viabilidad real para utilizar la magia como recurso didácticolúdico en el aula de 5 años de Educación Infantil.

2. Conocer qué tipo de habilidades propias de la creatividad se desarrollan con la magia como recurso educativo.

3. Comprobar la eficiencia de la magia como recurso educativo en el proceso de enseñanza-aprendizaje en los estudiantes de 5 años de educación infantil frente a otros métodos.

\subsection{Aspectos generales}


La metodología utilizada en este trabajo ha sido la investigación-acción (Login, 2004), ya que supone la búsqueda de información de forma continuada de forma que durante el proceso no solo se investiga sobre el caso que se pretende, sino que además se interviene de manera activa (Serrano, 1994). En nuestro caso se ha seguido el modelo en espiral en ciclos sucesivos incluyendo: a) Diagnóstico, b) Planificación, c) Acción, d) Observación y d) Reflexión (Bravo, 1994). La intervención se ha llevado a cabo con 20 alumnos de un aula de 5 años de un centro público de la provincia de Toledo, constituyendo el $50 \%$ del total de la población escolar en 5 años de esa localidad. Además, se ha contado con la colaboración 4 docentes del mismo centro y 10 expertosprofesionales del mundo de la magia.

\subsection{Instrumentos de investigación}

Los instrumentos de investigación utilizados han sido los siguientes:

- La rúbrica de evaluación en la observación directa y participativa, la cual ha servido para dar respuesta a los objetivos iniciales.

- La entrevista semiestructurada a expertos en magia y docentes en educación: la disposición de dos roles variados, en la que el elemento crucial va más allá de la simple interacción, porque uno de los sujetos manipula como a un objeto al otro (Fowler, 1990). En nuestra investigación, la entrevista ha tenido un formato semiestructurado, la cuál ha sido registrada en formato escrito y audiovisual. De igual modo tiene una intención exploratoria para dar una respuesta a los objetivos previos.

\subsection{Muestra}

\section{Profesionales de la magia}

Generalmente cada uno de los profesionales que han intervenido en la investigación disponen de unas capacidades y conocimientos sublimes, bien por su dedicación al mundo del ilusionismo, bien porque han adquirido una formación académica en lugares de prestigio como el Real Centro Universitario María Cristina de El Escorial o la escuela de magia Juan Tamariz. Su experiencia en el campo del ilusionismo ha sido fuente de riqueza para poder indagar de manera profunda en autores y en referencias bibliográficas, factores fundamentales para el análisis de dicha investigación. He aquí algunas de sus particularidades:

- La media de edad es de 43,3 años, algo que justifica el alto rodaje en el mundo del ilusionismo y la formación general que han adquirido y siguen adquiriendo cada uno de los profesionales.

- Su formación, tanto práctica como teórica, los llevan a formar parte del panorama internacional. Desde una perspectiva teórica, en el caso de Xuxo Ruíz, podemos comprobar que su publicación "Enseñando con magia" es el ejemplo de una metodología creativa e innovadora. No solo le sirve para desarrollar una enseñanza diferente e innovadora que motiva a los alumnos para aprender, sino que le lleva a conseguir el reconocimiento por parte de la Junta de la Comunidad de Andalucía con el premio al mérito educativo 2015. En varios casos, los investigados son autores de varias publicaciones relacionadas con la magia como un recurso educativo. 
Ejemplos de ello: "Educando con Magia", de Xuxo Ruíz como vimos anteriormente o "El abeZOOdario", de Javier Caboblanco. En cuanto a una perspectiva práctica, espectáculos donde la magia y la enseñanza están presentes son propios de Robín Ventura, con su espectáculo "Menudo Laboratorio", Alfonso V, con su intervención con Edelvives buscando la animación a la lectura o Fernando Arribas, que engloba la ciencia con el ilusionismo enfocado al mundo infantil con espectáculos titulados, "Astromagia", "magia conciencia" o "libros mágicos", este último dedicado a la animación a la lectura.

- Podemos comprobar que, en su mayoría, no solo desarrollan la magia mediante talleres, espectáculos o animaciones, sino que se dedican a la enseñanza de ésta en escuelas de magia. Es el caso de Rafael Pícolla, Alfonso V, Fernando Arribas o Xuxo Ruíz. En el caso del primero y el segundo, el objetivo es acercar a profesionales y/o aficionados al mundo de la magia, dándoles una formación teórica y práctica. En cuanto a los restantes, el acercamiento al mundo del ilusionismo se relaciona mayormente con aquellos que quieran emplear la magia en el aula con alumnos de Educación Primaria.

- Sus reconocimientos a nivel nacional o internacional demuestran la formación y la profesionalidad de cada uno de ellos.

\section{Docentes del centro escolar}

Antes de analizar las particularidades de los docentes hemos de anticipar que no desarrollan con normalidad este tipo de metodología. Vayamos pues a detallar sus características:

- La media de edad es de 46,7, un dato que nos ayuda a conocer cómo ha sido su formación académica y su experiencia en la docencia.

- Generalmente los docentes tienen una formación apta para participar en la investigación ya que han desarrollado talleres y cursos de formación que van acordes con el desarrollo de la creatividad, factor de dicha investigación.

- En todos los casos llevan a cabo cursos de formación relacionados con las TIC y las programaciones, con las artes plásticas y proyectos de innovación. Destacan los cursos y talleres de formación relacionados con la lectoescritura, con un aula creativa y expresiva, sobre elaboración de materiales en Educación Infantil o sobre cuentos infantiles que uno de los docentes ha impartido y sigue impartiendo para otros compañeros.

- En su mayoría acuden habitualmente a cursos de formación y seminarios relacionados con el desarrollo de la creatividad: métodos de enseñanza creativos como ABN (algoritmos basados en números), animación a la lectura, inteligencias múltiples, etc.) con el fin de enriquecer su carrera como docente.

- En el caso de un docente concreto, dispone de un blog digital donde expone actividades de creación propia y de otras fuentes relacionadas con la educación física, educación en valores, etc. 


\section{Análisis de los resultados}

La intervención se ha desarrollado en un aula de Educación Infantil de 5 años y situada en un CEIP de una población de la provincia de Toledo, y en la que han intervenido un total de 20 alumnos. Dicha intervención ha tenido como objetivo el desarrollo de la magia como recurso didáctico y lúdico para desarrollar la creatividad. La razón principal de esta elección no ha sido otra que la carga motivacional y lúdica que lleva en sí. Se puede decir que, por su atractivo, hace que el alumno esté predispuesto para el aprendizaje y que, por lo tanto, se sienta ilusionado (Olmedo, 2010). No obstante, el desarrollo de la curiosidad, la inquietud o la imaginación han sido factores que han impulsado la realización de este trabajo.

Pero hemos de señalar que, además del peso de la creatividad se han tenido en cuenta otras causas, quizá de igual o más importancia como son los factores relacionales. En Educación infantil no solo debe ser esencial el desarrollo de los conocimientos, al menos es lo que nuestra filosofía y metodología creativa e innovadora defendemos. Y por ello creemos que las actividades con magia pueden ser válidas para un buen desarrollo de las emociones, teniendo en cuenta su evolución psicológica (Vasta, 2001).

La pregunta más esperada por qué usar la magia como recurso educativo lleva su respuesta en la palabra magia, dada que es una palabra que cuando es pronunciada va acompañada de un gesto de sorpresa, de una actitud predispuesta a escuchar, de una motivación extra, en definitiva, de una ilusión por saber qué pasará.

Y dada su carga práctica y con la intención de comprobar si nuestros objetivos pueden tener el éxito esperado, consideramos que la intervención debe realizarse mediante la grabación, única forma de poder comprobar la intervención y ver cómo se desarrolla. Nos permite ver las reacciones de los alumnos, su interacción durante la actividad, los pros y los contras del proceso y cómo es el desarrollo del docente en el uso de la magia como recurso.

En el desarrollo normal de la intervención se han elaborado actividades grupales en su mayoría usando materiales, unos manipulados y otros elaborados a mano, relacionados con la magia infantil con el fin de enseñar los conceptos propios de una unidad didáctica que se estaba trabajando en el aula, centrada en el tema de las plantas. Por esta razón casi todas las actividades se engloban en este centro de interés. No obstante, han estado presentes otras temáticas como la educación vial, los animales o los cuentos en valores usando la magia como herramienta de aprendizaje.

Estas actividades han sido evaluadas mediante los registros correspondientes propios de la unidad didáctica, todo ello a través de escalas de observación o mediante items según establece el real decreto 67/2007 de segundo ciclo de Educación Infantil.

\subsection{Rasgos generalizados}

Teniendo en cuenta el objeto de estudio, la magia como recurso didáctico-lúdico para el desarrollo de la creatividad en alumnos de 5 años de Educación infantil, analizamos y damos respuesta a cada una de las preguntas formuladas: 
A) Existe viabilidad real para utilizar la magia como recurso didáctico-lúdico en el aula de 5 años de Educación Infantil. La respuesta de esta primera cuestión tiene un carácter positivo y en todas las variables coincide en la viabilidad de la magia como un recurso didáctico-lúdico para un aula de 5 años de Educación Infantil, ya que:

1. La magia capta la atención de los alumnos. Es la respuesta más común de los entrevistados, tanto por los profesionales de la magia como por los docentes del centro escolar. La magia es un recurso que motiva a los alumnos y les hace fijar su atención con más facilidad que con otro recurso evitando, por lo tanto, distracciones y dando lugar a un aprendizaje mejor. Con esa actitud de predisposición los alumnos comprenden mejor lo que han aprendido. De igual manera podemos conseguir que pierda la vergüenza a hablar en público.

2. La magia lleva impregnado el aprendizaje. Destacamos que la magia, a diferencia que otras materias, lleva en sí una carga de aprendizaje de forma más emocional y memorable. Podemos desarrollar desde otro enfoque factores como: la curiosidad, las habilidades artísticas o la comunicación.

3. Está dentro de una metodología distinta al resto. Algo que caracteriza al uso de la magia como un recurso de aprendizaje, como afirman los profesionales, es que enseñar con magia es algo nuevo para los alumnos que parte de sus intereses y que, por lo tanto, no viene en los libros. Esta metodología incluye enseñar las áreas del currículo de Educación Infantil de manera divertida y motivadora.

4. La magia absorbe y motiva al alumno. Es un arte ilusionante. Educación Infantil es una etapa de pleno desarrollo donde el alumno está predispuesto a aprender aquello que se le enseña. Es considerada la etapa de "esponja", por lo tanto, podemos aprovecharla para el aprendizaje de cualquier contenido. Su ilusión por conocer cosas nuevas, por explorar y experimentar es propia de la magia. No debemos condicionar a los alumnos a que hagan algo que para nosotros es algo "común" sino dejar que sean ellos mismos los que digan lo que quieren aprender.

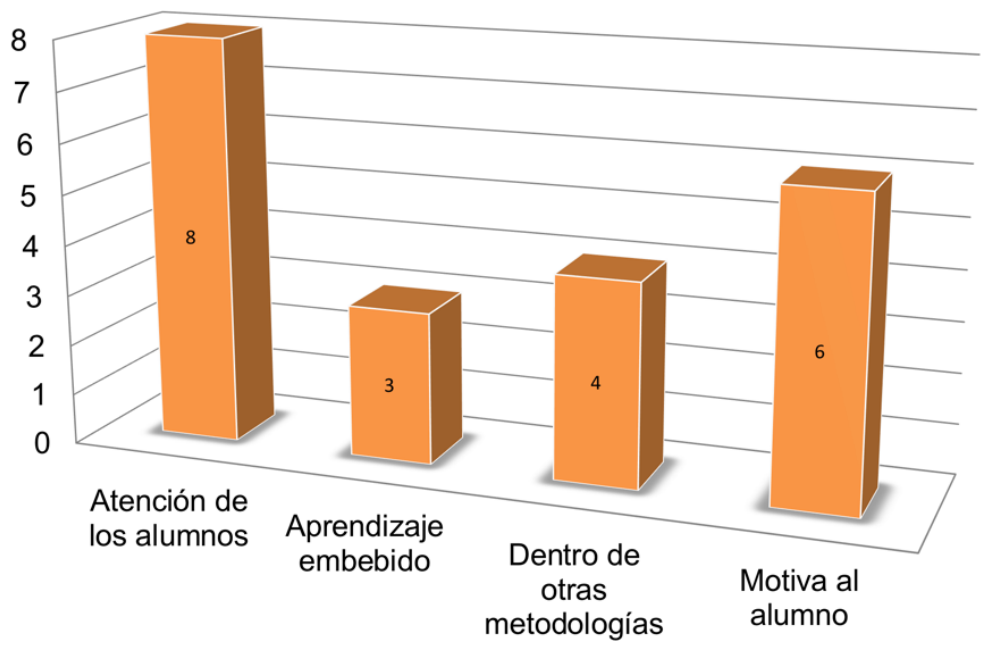


Gráfico 1. Viabilidad como recurso didáctico-lúdico entre profesionales y docentes.

x: característica de viabilidad; y: coincidencias entre los entrevistados. Fuente: elaboración propia

B) Las niñas y niños de 5 años desarrollan habilidades propias de la creatividad a través de la magia como recurso educativo. Podemos comprobar cómo coinciden en su mayoría que la magia es una herramienta muy beneficiosa y útil para desarrollar la creatividad, ya que:

1. La magia ayuda a desarrollar habilidades de comunicación. Las habilidades comunicativas, tanto la escucha como el habla, en el caso del uso de la magia como una herramienta, son habilidades que un alumno emplea de forma común. En el caso de los profesionales que se refieren al alumno como mago, afirman que la comunicación implica la adquisición de aspectos relacionados con la creatividad: la libertad de expresión, saber esperar, respetar la palabra, aprender a hablar en público con fluidez y sin monotonía, no tener miedo a equivocarse, ser él mismo, desarrollar las habilidades narrativas y expresivas, etc. En el caso del uso de la magia por parte del docente para explicar cualquier contenido o por simple diversión, el alumno, en este caso, desarrolla otras habilidades relacionadas con la comunicación: atención, escucha activa, comprensión, etc.

2. La magia desarrolla las habilidades artísticas y creativas. Varios son los que coinciden en que la magia favorece al desarrollo de las habilidades manipulativas y artísticas. Si el alumno es partícipe de las actividades mágicas tendrá que elaborar, mediante materiales, el juego de magia; de manera que las habilidades tienen un sentido mayor, pero si, por otra parte, forman parte de la actividad como espectador, esas habilidades tendrán un carácter más creativo como: imaginación, curiosidad, inquietud, etc.

3. La magia ayuda a relacionarnos con los demás. Como bien dicen los profesionales la magia tiene una función socializadora. Podemos comprobar que se establecen vínculos mayores entre los iguales cuando se trabaja la magia y por lo tanto se relacionan y dialogan entre ellos de manera positiva.

4. La magia es producto de nuestra imaginación. Es uno de los factores más importantes e ilusionantes de emplear la magia como una herramienta de aprendizaje. El desarrollo de la imaginación es el más usado por el alumno cuando forma parte de manera activa del juego, así pues, tiende a imaginase lo que no ve, a ilusionarse, a sorprenderse, a crear hipótesis, a hacer aproximaciones, a inventarse respuestas, a realizar preguntas, etc. 


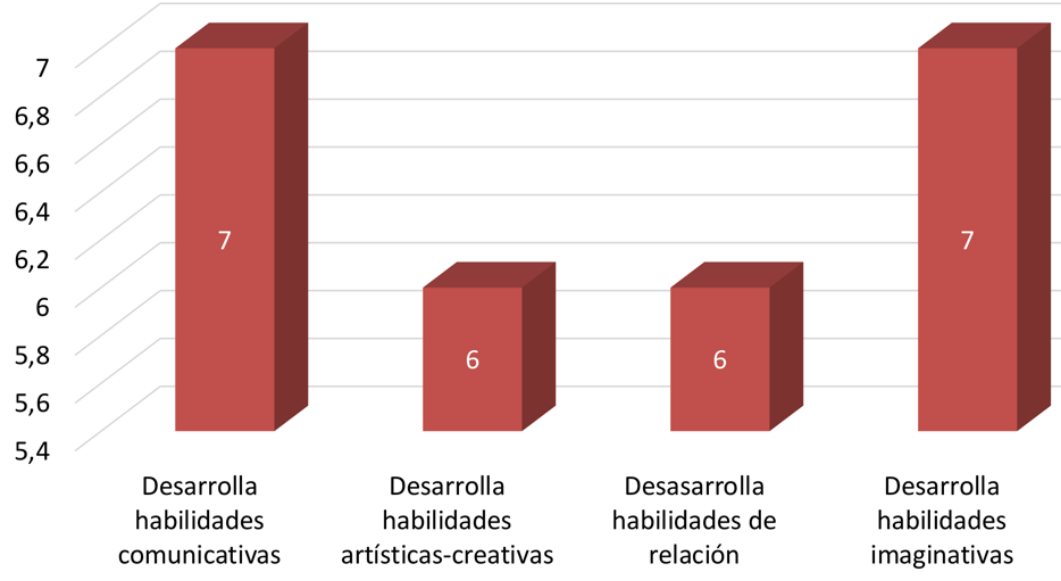

Gráfico 2. Habilidades desarrolladas con la magia, entre profesionales y docentes

$\mathrm{x}$ : habilidades desarrolladas; $\mathrm{y}$ : coincidencias entre los entrevistados. Fuente: elaboración propia

C) Existe una eficiencia de la magia, como recurso educativo, en el proceso de enseñanza-aprendizaje en los estudiantes de 5 años de educación infantil frente a otros métodos. En cualquiera de las entrevistas se defiende el uso de la magia como recurso porque se basa en la creatividad, es diferente a las demás, ilusionante, divertida e interesante y porque parte de los intereses del alumno sin dejar de lado otros recursos, por varias razones como:

1. La magia es una herramienta como cualquier otra. Muchos maestros afirman que la magia no es, en muchos de los casos, considerada como una herramienta de aprendizaje sino como una forma de entretener. Los profesionales investigados defienden que la magia debe ser usada para enseñar al igual que la música, el teatro o el guiñol y que no hemos ni restarle la importancia ni sobrevalorarla.

2. Un maestro debe tener una bolsa llena de recursos. Sin darle más importancia a la magia que al resto, aclaran que todos los recursos tienen que estar unidos y que uno favorezca al otro. Un maestro debe disponer de una bolsa con muchos recursos y en el momento de necesitarlo cogerlo. Es decir, debe ser polivalente, pudiendo ser mago, payaso, músico, cantante o bailarín.

3. La magia forma parte de una metodología innovadora. No dudan en ningún caso del tipo de metodología de la que forma parte este tipo recurso. La engloban como una metodología innovadora, o en algún caso, en pañales (que no está estudiada), diferente al resto, atractiva y útil. Defienden de igual forma que debe ser complementaria y usarla en los momentos necesarios.

4. La magia engloba ilusión, innovación y creatividad. Esta herramienta atractiva y eficaz es una ventaja para un maestro, y aquel que tiene esa oportunidad dispone de armas para desarrollar un aprendizaje mejor. No solo conseguir la diversión del alumno sino lograr en él la predisposición para ir a clase. Esta herramienta va acorde con la ilusión y el mundo en el que está el alumno, es decir, un mundo de fantasía o de imaginación. 


\section{Conclusiones}

A partir del análisis de los datos obtenidos, podemos evidenciar que, los objetivos de investigación han sido validados. Partiendo de la aprobación de cada uno estos objetivos hemos llegado a las siguientes conclusiones:

La magia es practicable con alumnos de 5 años de Educación Infantil como un recurso didáctico y lúdico. La magia como recurso está incluida en una metodología activa que orienta a los alumnos a conseguir sus aprendizajes, guiarles y ayudarles a desarrollar su personalidad (Freinet, 1973). Evidentemente el desarrollo de la magia en un aula de Educación infantil es factible. Como bien decía Ciuró (2007) proporciona unas habilidades, unas destrezas y un ingenio tanto por parte de la persona que lo desarrolla como por la persona que participa de ella. Su motivación acerca al alumno en una actitud activa y positiva hacia el aprendizaje. Lleva inmersa una carga lúdica que hace que el disfrute y aprendizaje se mantengan unidos durante toda la actividad y, por consiguiente, active su atención y predisposición hacia el docente (Gértrudix \& Gértrudix, 2013).

La magia favorece al desarrollo de la creatividad. Como bien afirma Robinson (2011) la creatividad consiste en generar ideas originales. Pues bien, se ha comprobado que la magia en un aula de Educación Infantil actúa de una forma similar. Forma parte de cualquiera de los tipos de creatividad que hemos visto; expresiva, productiva, inventiva, innovadora y emergente (Sanz de Acedo Lizárraga y Sanz de Acedo Baquedano, 2007). Ya lo anticipa Amegan (1993) cuando nos ofrece los tipos de actividad creativa. En el caso de un aula de 5 años es una actividad creativa dado que el alumno goza de una libertad de elección de los medios y objetivos, que es una actividad efectiva ya que saca lo mejor de ellos y es una actividad de fabricación porque tiene las características necesarias para que el alumno logre un objetivo. La creatividad incluye el desarrollo de los rasgos motivacionales, cognitivos y socioculturales, comprobando que la magia cumple con cada uno esos rasgos mencionados.

La magia es producto de nuestra imaginación. La imaginación es una de las habilidades que más desarrolla el alumno cuando ve un juego de magia, es capaz de generar ideas originales, de pensar en cosas que no están presentes o, incluso, elementos que no existen. Ponen a trabajar sus sentidos y relucir la inquietud, la originalidad, las habilidades cognitivas, la astucia y el razonamiento (Robinson, 2011). El niño experimenta una etapa donde el juego simbólico forma parte activa en su vida, donde todo lo que se le muestra piensa que existe.

La magia forma parte de una enseñanza creativa e innovadora. Una metodología usando la magia como recurso, ofrece de forma clara y directa una enseñanza abierta y flexible donde los alumnos se inquietan por saber lo que va a pasar, lo que pasa o lo 
que ha pasado, ya sea en las matemáticas (matemagia), en la ciencia o en los juegos de desapariciones (Wiliams, 1970). Posibilita que el alumno se planteé hipótesis, pueda sacar sus propias conclusiones o realice preguntas que le lleven a ser curioso e investigador. Esta metodología es creativa por varias razones; 1) igualdad en el proceso y la acción, 2) estimula el pensamiento divergente, 3) reduce la tensión de los alumnos, 4) promueve los conocimientos, 5) impulsa a la manipulación y exploración, 6) utiliza métodos para un pensamiento creativo, 7) incita a los alumnos a generar ideas originales, porque alienta la critica constructivista, 8) permite experiencias personales de los alumnos, 9) porque utiliza situaciones abiertas de aprendizaje y 10) se centra en el éxito y no en el fracaso (Smith, 1970). La finalidad será conseguir la participación del estudiante para que este tipo de estrategias puedan tener cabida en su vida cotidiana.

A pesar de los positivos resultados obtenidos, debemos ser conscientes que el trabajo de campo se ha realizado como estudio de caso, y por lo tanto los datos están referidos a un grupo concreto de alumnos, con una edad y un lugar determinados. Es por ello, que dichos resultados serían diferentes si propusiéramos la investigación para otro tipo de alumnado, nivel, centro escolar o distinta localidad. Es decir, el contexto donde se desenvuelve la investigación está relacionado con los resultados que se obtienen y es muy determinante para obtener unas conclusiones concretas, así como los recursos de los que disponemos para desarrollar la magia, ya que se requiere de unos conocimientos y formación definidos, así como tener acceso a unos materiales específicos. En este trabajo se ha tenido la oportunidad de contar con ambos factores al alcance. Pero es bien cierto que este tipo de recursos, como dice Rafael Pícolla: "está en pañales"; es decir, no está estudiado y es poco habitual su desarrollo en un aula de Educación Infantil.

Así pues, han surgido nuevas líneas de investigación que en un futuro se podrían trabajar y, gracias a los datos obtenidos por los expertos, se ha comprobado que la magia sí podría utilizarse en educación infantil.

Por lo tanto, y, como síntesis a nuestra investigación, resaltar que la magia proporciona muchas posibilidades de investigación desde muchos enfoques y muchas perspectivas porque como dice Xuxo Ruíz, si el significado de magisterio es la actividad del maestro y las enseñanzas que transmite a sus alumnos, podríamos cambiar esa palabra por magisterio, actividad del maestro y las enseñanzas que transmite a sus alumnos mediante la magia.

\section{Bibliografía}

\section{PUBLICACIONES PERIÓDICAS}

Engle, D. M. (1997). An empirical comparison of entrepreneurs and employees: Implications for innovation. Creativity Research Journal, 10(1), 45-49 
Gértrudix Barrio, F., y Gértrudix Barrio, M. (2013). Aprender jugando. Mundos inmersivos abiertos como espacios de aprendizaje de los y las jóvenes. Revista de Estudios de juventud, 101, 123-137.

Newell, A. S. (1962). The process of creative thinking In H. Gruber.G.Terrell. \& M. Wertheimer. Contemporary approaches to creative thinking. New York: Atherton, pp. 63-119. doi:10.1037/13117-003

Olmedo, P. (2010). Implicaciones del desarrollo cognitivo en el proceso de enseñanzaaprendizaje en el alumno de 6 a 16 años. Revista de Psiquiatría y Psicología del niño y adolescente, 9(1), $1-10$

Otero, J. M. (2000). Más allá de las ideas previas como dificultades de aprendizaje: Las pautas de pensamiento. Las concepciones epistemológicas y las estrategias metacognitivas de los alumnos de ciencias. Enseñanza de las ciencias: revista de investigación u experiencias didácticas, 18(2), 156-166.

Sternberg, R. J. (1997). The concept of intelligence and its role in lifelong learning and success. American Psychologist, 52(10), 1030-1037. http://dx.doi.org/10.1037/0003066X.52.10.1030

Vidal, J. M. (2009). La importancia de la socialización en la Educación actual. Innovación y experiencias educativas, $14,2-9$.

\section{LIBROS Y CAPÍTULOS DE LIBRO}

Amegan, S. (1993). Para una pedagogía activa y creativa. México: Trillas.

Bransford, J. S. (1984). The ideal problem solver: A guide for improving thinking learning, and creativity. New York: Freeman.

Bravo, M. P. (1994). Investigación Educativa. La investigación-acción. Sevilla: Alfar.

Codoni, M. (1987). Metodología activa: reflexiones y experiencias. Madrid: Acción educativa D.L.

Davis, G. (1983). Creativity is forever. lowa: Kendall/Hunt.

Denzin, N. (1970). Sociological Methods. A sourcebook. Chicago, IL: Aldine Publishing Company.

Fowler, F. J. (1990). Standardized Survey Interviewing-Related Error. Newbury park: Sage.

Freinet, C. (1973). Técnicas Freinet de la Escuela Moderna. Madrid: Siglo XXI.

Goleman, D. (1995). Inteligencia Emocional. Kairós S.L. 
Paquette, C. (1976). Vers une practique de la pèdagogie ouverte. Victoriaville, Quebec: Editions NHP.

Rousseau, J. (1978). El Emilio. Madrid: Edaf.

Ruiz, X. (2013). Educando con magia. El ilusionismo como recurso didáctico. Madrid: Narcea, S.A. de Ediciones.

Sanz de Acedo Lizárraga, M. S. y Sanz de Acedo Baquedano, M.T. (2007). Creatividad individual y grupal en la educación. Madrid: Ediciones Internacionales Universitarias, Eiunsa, S.A.

Serrano, G. P. (1994). Investigación cualitativa. Retos e interrogantes (6ª ed.). Madrid: La Muralla.

Smith, J. A. (1970). Setting Conditions of creative Teaching in the Elementary School. Boston: Allyn and Bacon.

Storr, A. (1988). Solicitude: a return to the self. New York: The Free Press.

Vasta, R. H. (2001). Psicología Infantil. Barcelona.

Williams, F. E. (1970). Classroom Ideas for Encouraging Thinking and Feeling. Búfalo, Nueva York: D.O.K. Publishers.

\section{MEDIOS ELECTRÓNICOS}

EDUCACIÓN 3.0 (22 de julio de 2014). Informagia, trabajar las competencias a través de la magia. Obtenido de https://goo.gl/eplJDp

Escuela de Magia Alameda (2014). Escuela de Magia Alameda. Obtenido de: http://magiaalameda.wixsite.com/informagia

Greig, J. (18 de mayo de 2016). Why Educational Magic Is Perfect for the 21st-Century Classroom and Entertainer. KidsEntertainerHub.com. Obtenido de: https://goo.gl/h8UjTA

Kehl, W. (6 de enero de 2014). 8 Resources for Teaching STEM with Magic. Learning, online \& blended, prek-12. Obtenido de: http://gettingsmart.com/2014/01/8-resources-teachingstem-magic/

Login, N. A. (26 de enero de 2004). La investigación Acción Crítica Reflexiva. Obtenido de MasEducativa, 5: http://goo.gl/f04ZoQ

Martín, S. (1 de febrero de 2011). Por arte de magia. Aula del Pedagogo. Obtenido de: http://www.auladelpedagogo.com/2011/02/por-arte-de-magia/ 
Robinson, K. (13 de enero de 2011). Las escuelas matan la creatividad. Obtenido de TED: Recuperado de: https://www.youtube.com/watch?v=AW-bTuBA5rU

Simó, R. (2014). Aplicar la magia en la escuela: propuesta de intervención en la educación formal. TFG. Salamanca: Repositorio Documental de la Universidad de Salamanca. Obtenido de: http://gredos.usal.es/jspui/handle/10366/125855

\section{REFERENCIA BIBLIOGRÁFICA}

López González, D. y Gértrudix-Barrio, F. (2019). La magia como recurso didáctico-lúdico para el desarrollo de la creatividad en niñas y niños de 5 años. Aula de Encuentro, n²1 (1), pp. 143-159.

David López González es Profesor de Educación Infantil e Investigador colaborador del Grupo de Investigación CIBERIMAGINARIO-UCLM de la Universidad de Castilla-La Mancha Correo-e: pichelin33@gmail.com

Felipe Gértrudix-Barrio es Profesor Titular de Universidad e IP del Grupo de Investigación CIBERIMAGINARIO-UCLM de la Universidad de Castilla-La Mancha Correo-e: felipe.gertrudix@uclm.es 